\title{
ARTICLES
}

\section{CCL2 Induces Prostate Cancer Transendothelial Cell Migration Via Activation of the Small GTPase Rac}

\author{
Kenneth L. van Golen, ${ }^{1,2}$ Chi Ying, ${ }^{3}$ Linda Sequeira, ${ }^{1,2}$ Cara W. Dubyk, ${ }^{1}$ Tracy Reisenberger, ${ }^{1}$ \\ Arul M. Chinnaiyan, ${ }^{4}$ Kenneth J. Pienta, ${ }^{3,5}$ and Robert D. Loberg ${ }^{3,5 *}$ \\ ${ }^{1}$ Department of Biological Science, Laboratory of Cytoskeletal Physiology, University of Delaware, \\ Newark, Delaware \\ ${ }^{2}$ The Center for Translational Cancer Research, Newark, Delaware \\ ${ }^{3}$ Department of Internal Medicine, University of Michigan, Ann Arbor, Michigan \\ ${ }^{4}$ Department of Pathology, University of Michigan, Ann Arbor, Michigan \\ ${ }^{5}$ Department of Urology, University of Michigan Urology Center, Ann Arbor, Michigan
}

\begin{abstract}
Nearly $85 \%$ of the men who will die of prostate cancer (PCa) have skeletal metastases present. The ability of PCa cells to interact with the microenvironment determines the success of the tumor cell to form metastatic lesions. The ability to bind to human bone marrow endothelial (HBME) cells and undergo transendothelial cell migration are key steps in allowing the PCa cell to extravasate from the bone microvasculature and invade the bone stroma. We have previously demonstrated that monoctyte chemoattractant protein 1 (MCP-1; CCL2) is expressed by HBME cells and promotes PCa proliferation and migration. In the current study, we demonstrate that the CCL2 stimulation of PCa cells activates the small GTPase, Rac through the actin-associated protein PCNT1. Activation of Rac GTPase is accompanied by morphologic changes and the ability of the cells to undergo diapedesis through HBME cells. These data suggest a role for HBMEsecreted CCL2 in promoting PCa cell extravasation into the bone microenvironment. J. Cell. Biochem. 104: 1587-1597, 2008. (c) 2008 Wiley-Liss, Inc.
\end{abstract}

Key words: actin cytoskeleton; diapedesis; monoctye chemoattractant protein 1; metastasis

Prostate cancer $(\mathrm{PCa})$ skeletal metastasis is a major clinical concern associated with intractable pain, bone fracture, and paralysis resulting from spinal cord compression and a rapid degradation in quality of life [Thalmann et al., 2000]. Of the more than 30,000 men that will die

Abbreviations used: PCa, prostate cancer; Rho, Ras Homolgy; HBME, human bone marrow endothelial cells; PCNT1, pericentrin; CCL2, monocyte chemoattractant protein-1; CCR2, CCL2 receptor.

Grant sponsor: University of Michigan Prostate SPORE Career Development Award; Grant numbers: P50 CA69568-06A, PO1 CA093900-01, CDMRP PCRP W81XH04-0225, W81XH-05-0005; Grant sponsor: The University of Delaware/Delaware Biotechnology Institute Start Up Funds.

*Correspondence to: Dr. Robert D. Loberg, PhD, University of Michigan Urology Center, 7411 CCGC, 1500 E. Medical Center Dr., Ann Arbor, MI 48109-0946.

E-mail: rloberg@umich.edu

Received 10 October 2007; Accepted 2 November 2007

DOI 10.1002/jcb.21652

(c) 2008 Wiley-Liss, Inc. of PCa in 2007, nearly $85 \%$ will die with significant skeletal metastases and associated co-morbidities [Thalmann et al., 2000].

The metastatic cascade is an ordered series of steps that a cancer cell must successfully complete to form a tumor at a distant site [Fidler, 1990]. One key step is extravasation from the circulation to the distant organ environment [Fidler, 1990]. PCa cells undergo transendothelial cell migration (tumor cell diapedesis) across a barrier of bone marrow endothelial cells to extravasate into the bone microenvironment [Lehr and Pienta, 1998; Cooper and Pienta, 2000; Cooper et al., 2000]. The molecular mechanisms involved in $\mathrm{PCa}$ tumor cell diapedesis have not been extensively explored.

Recently, we have reported a role for the Rho GTPases in PCa bone metastasis [Yao et al., 2006]. Rho GTPases are molecular switches involved in reorganizing the actin cytoskeleton and are key mediators of metastasis [Ridley and Hall, 1992b; Esteve et al., 1998; Gampel et al., 1999; Evers et al., 2000; Sahai and Marshall, 
2002; Hall et al., 2006]. Previously, we have demonstrated a role for RhoC and Rac GTPases in $\mathrm{PCa}$ cell migration and invasion in response to bone-associated factors, implicating these proteins in the process of bone metastasis [Hall et al., 2006; Yao et al., 2006]. Our data demonstrate specific roles for the individual GTPases in PCa cell invasion and transendothelial cell migration, suggesting that activation of differential GTPase activation controls these events.

Chemotaxis and motility are essential components of tumor cell trafficking and metastasis [Romanov and Goligorsky, 1999]. Therefore, identifying the key components linking the connection between the chemoattractant-activated receptor and cytoskeletal reorganization is an important part of understanding tumor biology and may lead to the identification of novel therapeutic targets. Previously, we demonstrated that human bone marrow endothelial (HBME) cells secrete high levels of CCL2, a member of the CC $\beta$ chemokine family primarily known to promote monocyte and macrophage migration to the sites of inflammation [Ohta et al., 2002; Balkwill, 2003; Loberg et al., 2006]. CCL2 acts as a mitogenic factor for PCa cells, but also stimulates cellular motility and may act to recruit $\mathrm{PCa}$ cells to the bone microenvironment [Loberg et al., 2006; Loberg et al., 2007].

Like other chemokines, CCL2 potentially regulates tumor cell motility through the activation of the Rho GTPases [van Golen, 2003]. This is highly likely since CCL2-stimulated monocyte chemotaxis is linked to the actin cytoskeleton via the interactions of the putative CCR2 receptor with PCNT1, a novel actin regulating protein [Terashima et al., 2005]. Stimulation of CCR2 with its ligand, CCL2, induces clustering of the receptor and PCNT1 on the leading edge of monocytes and macrophages [Terashima et al., 2005] and regulates the ability of these cells to migrate through activation of Rac GTPase. Activation of Rac GTPase is required for lamelipodia formation, which in turn is required for sensing the junctions between the endothelial cells [Terashima et al., 2005]. Previously, we demonstrated that CCL2 stimulation of PC-3 PCa cells led to distinct changes in cellular morphology [Loberg et al., 2006]. The morphological changes were consistent with the epithelial to mesenchymal transition (EMT) observed when RhoC GTPase activity was downregulated in
PC-3 cells [Yao et al., 2006]. The observed EMT was accompanied by an increase in sustained Rac GTPase activity [Yao et al., 2006]. In the current study, we hypothesized that activation of the canonical CCL2-CCR-PCNT1 cascade activates Rac GTPase thus inducing morphological changes and tumor cell diapedesis. Our data demonstrate that CCL2 activation of PCNT1 increases total and active levels of Rac GTPase, which is required for PC-3 cell binding and diapedesis through HBME cells. These data give insights to the molecular mechanisms potentially involved in PCa extravasation from the bone microvasculature.

\section{MATERIALS AND METHODS}

\section{Cells and Cell Culture and Reagents}

PC-3 PCa cells, originally derived from a human bone metastasis, was obtained from ATCC (Mannassass, VA). Human bone marrow endothelial (TrHBMEC) cells were a kind gift from Babette Weksler, Cornell University [Schweitzer et al., 1997]. PC-3 cells were maintained in $10 \%$ fetal bovine serum (Gibco), 90\% Ham's F-12 (Invitrogen Corp., Carlsbad, CA) growth medium at $37^{\circ} \mathrm{C}$ in $5 \% \quad \mathrm{CO}_{2}$. TrHBMECs, from here out termed HBME cells, were grown in $10 \% \mathrm{FBS}$ and $90 \% \mathrm{DMEM}$ at $37^{\circ} \mathrm{C}$ in $5 \% \mathrm{CO}_{2}$.

\section{PCNT1 and Rac siRNA}

PCNT1 siRNA constructs were purchased from Ambion, Inc. (Austin, TX) and Rac-specfic Smart-pools siRNA was purchased from Upstate Biotechnology (Charlottesville, VA). For transient transfection of PC-3 cells $10 \mu \mathrm{g}$ siRNA or scrambled sequence was introduced into actively growing PC-3 cells at $40 \%$ confluence in 35-mm dishes using Fugene6 transfection reagent (Roche, Indianapolis, IN). Efficiency of siRNA-mediated downregulation of PCNT1 and Rac was tested $72 \mathrm{~h}$ after introduction of the siRNA by Western blot analysis. Previously siRNA targeting of Rac was shown to decrease Rac protein levels by 7580\% [Lin et al., 2005]. PCNT1 downregulation was confirmed by Western blot analysis using an anti-PCNT1 antibody (Abcam, Inc.) using standard SDS-PAGE under reducing conditions. All experiments involving siRNA were conducted $72 \mathrm{~h}$ after introduction of the siRNA into the cells. Matched scrambled controls were used for both the PCNT1 and Rac siRNAs. 


\section{cDNA Microarray}

Two PCa bone metastases and a soft tissue (adrenal) metastasis were collected from each of three patients from the rapid autopsy program at the University of Michigan. Tissue specimens were verified for purity by a pathologist and compared at the transcriptome level by cDNA microarray analysis comprised of $20 \mathrm{~K}$ cDNA clones. Microarrays were hybridized and analyzed as previously described [Xin et al., 2003]. Briefly, RNA from the PCa specimens was used to synthesize labeled cDNA (Cy5) prior to hybridization and analysis. Microarray slides were processed and scanned with a Genepix 4000B scanner (Axon Instruments, Union City, $\mathrm{CA})$.

\section{Real Time (RT)-PCR}

Total RNA was isolated from cell lines using Trizol (Invitrogen Corp.) following the manufacturer's specifications. Purified RNA (5 $\mu \mathrm{g}$ ) was converted to cDNA using Super Script II reverse transcriptase (Invitrogen Corp.) following the manufacturer's instructions and used for gene expression analysis by RT-PCR using an ABI Prism 7900 HT thermocycler. Primers and probes were purchased from Applied Biosystems, Inc. and used with TaqMan ${ }^{\circledR}$ Universal PCR Master Mix, No AmpErase ${ }^{\circledR}$ UNG. GAPDH was used as an internal control to normalize and compare each sample. Cycle conditions for RT-PCR were $95^{\circ} \mathrm{C}(15 \mathrm{~s}), 60^{\circ} \mathrm{C}$ ( $1 \mathrm{~min}$ ), and $72^{\circ} \mathrm{C}(1 \mathrm{~min})$ for 40 cycles. Threshold cycle number for each sample was normalized to GAPDH for that sample and expressed on a log scale relative to GAPDH expression.

\section{Immunofluorescence}

PC-3 cells were plated on glass coverslips and were stimulated with $100 \mathrm{ng} / \mathrm{ml}$ recombinant human CCL2 (Chemicon Inc.) for $24 \mathrm{~h}$ in the presence or absence of identified inhibitors. Cells were fixed in $3.7 \%$ paraformaldehyde, methanol-free for $10 \mathrm{~min}$ at room temperature then permeablized with $0.5 \%$ Triton X-100 for $5 \mathrm{~min}$ at room temperature. Cells were rinsed twice with PBS and incubated for $30 \mathrm{~min}$ at room temperature with $3 \%$ bovine serum albumin (BSA) in PBS $+0.05 \%$ Tween20 to prepare cells for staining. Cells were incubated with anti-PCNT1 (Abcam, Inc.) at a 1:50 dilution in BSA solution followed by donkey anti-goat AlexaFluor 488 secondary antibody at 1:100 dilution (Molecular Probes, Inc.). Cells were costained with Rhodamine-phalloidin (Molecular Probes, Inc.). Cells were washed and mounted on coverslips with Pro-Long Antifade containing DAPI (Molecular Probes, Inc.) following manufacturer's instructions. Immunofluoresence was visualized using an Olympus IX71 inverted microscope.

\section{Rac GTPase Activation Assays and Western Blot Analysis}

The PAK binding assays were performed as previously described [Hall, 1990]. Briefly, Escherichia coli BL21 cells transformed with the glutathione s-transferase (GST)-PAK-CD (pAK-CRIB domain) construct (a generous gift from Dr. John Collard of The Netherlands Cancer Institute) were grown at $37^{\circ} \mathrm{C}$ overnight. Expression of recombinant protein was induced with $100 \mu \mathrm{M}$ isopropylthiogalactoside (Sigma Chemical Co., St. Louis, MO) for $2 \mathrm{~h}$. Cells were pelleted and resuspended in lysis buffer (50 mM Tris-HCl, pH 8.0, $2 \mathrm{mM} \mathrm{MgCl}_{2}$, $200 \mu \mathrm{M} \mathrm{Na} \mathrm{Na}_{2} \mathrm{O}, 10 \%$ glycerol, $20 \%$ sucrose, $2 \mathrm{mM}$ dithiothreitol, $1 \mathrm{mM}$ benzamide, $1 \mathrm{mM}$ PMSF, $1 \mu \mathrm{g} / \mathrm{ml}$ leupeptin, $1 \mu \mathrm{g} / \mathrm{ml}$ pepstatin, and $1 \mu \mathrm{g} / \mathrm{ml}$ aprotinin). After sonication, cell lysates were centrifuged at $4^{\circ} \mathrm{C}$ for $20 \mathrm{~min}$ at $45,000 \mathrm{~g}$ and supernatant was incubated with glutathione-coupled sepharose 4B beads (Amersham Biosciences Corp., Piscataway, NJ) for $30 \mathrm{~min}$ at $4^{\circ} \mathrm{C}$. Protein bound beads were washed three times in lysis buffer and resuspended in GST-FISH buffer (50 mM Tris, pH 7.4, $2 \mathrm{mM}$ $\mathrm{MgCl}_{2}, 100 \mathrm{mM} \mathrm{NaCl}, 10 \%$ glycerol, $1 \%$ NP-40, $1 \mathrm{mM}$ benzamide, $1 \mathrm{mM}$ PMSF $1 \mu \mathrm{g} / \mathrm{ml}$ leupeptin, $1 \mu \mathrm{g} / \mathrm{ml}$ pepstatin, and $1 \mu \mathrm{g} / \mathrm{ml}$ aprotinin). PC-3 cells were starved for $2 \mathrm{~h}$, stimulated with $100 \mathrm{ng} / \mathrm{ml}$ recombinant human CCL2 for $30 \mathrm{~min}$, washed three times with ice-cold PBS, and lysed in GST-FISH buffer. Protein concentration in the supernatant was determined by Biorad protein determination dye (Bio-Rad Laboratories, Hercules, CA). Supernatant (1 mg) was incubated with glutathione-sepharose GSTfusion protein beads for $30 \mathrm{~min}$ at $4^{\circ} \mathrm{C}$. Pull down beads were washed three times in GSTFISH buffer, resuspended in Laemmli buffer and analyzed by Western blotting using antiRac (Upstate USA, Inc., Charlottesville, VA) monocolonal antibody. Bands were visualized by ECL chemiluminescence (Promega Corp., Madison, WI) and autoradiographic intensities were quantitated using ImageJ software (NCI, 
Bethesda, MD). Western blot analysis for total GDP- and GTP-bound Rac GTPase was performed as previously described [Yao et al., 2006].

Quantitative analysis of Rac activation was performed using the GLISA Rac activation assay (Cytoskeleton, Inc., Denver, CO). Briefly, PC-3 cells were grown to $50 \%$ confluence and transfected with $10 \mu$ g pooled PCNT1 siRNA or scrambled control using FuGene6. Cell lysates were prepared $72 \mathrm{~h}$ later and normalized according to the manufactures specifications. Protein concentrations of $2 \mathrm{mg} / \mathrm{ml}$ and $1 \mathrm{mg} / \mathrm{ml}$ were used to determine Rac activity using a 1:250 dilution of the primary antibody and 1:250 dilution of the secondary antibody. After antibody and horse radish peroxidase detection reagent incubation, signals were detected on a Dynex MLX microplate spectrophotometer. Data analysis was performed on GraphPad Prism 4.01.

\section{Diapedesis}

HBME cells $(10,000$ cells $/ \mathrm{ml}-0.5 \mathrm{ml})$ were layered onto a Matrigel ${ }^{\mathrm{TM}}$ coated filter (24 well plate, $8 \mu \mathrm{m}$ pore) and grown to confluence $(22 \pm 2 \mathrm{~h})$. The day prior to the experiment the PC-3 cells were grown in 6-well plate to $50 \%$ confluence. Cells were transfected with scrambled control or siRNA to PCNT1 or Rac GTPase (Upstate Biotechnology) using $\mathrm{Fu}$ Gene6 (Roche). siRNA-treated cells were seeded $\left(2.75 \times 10^{5} / \mathrm{ml}, 0.5 \mathrm{ml}\right)$ onto the HBMEs at $48 \mathrm{~h}$ after transfection in serum free medium. The lower chamber contained serum (10\% FBS) containing growth medium. The cells were allowed to invade for $24 \mathrm{~h}$ (therefore $72 \mathrm{~h}$ post siRNA-treatment), the upper chamber was aspirated and non-invaded cells and Matrigel ${ }^{\mathrm{TM}}$ wiped away with a Q-tip. The filter was stained with $0.1 \%$ crystal violet in ethanol for $10 \mathrm{~min}$, rinsed $3 \times$ with water and air-dried. The cells that were attached to the bottom of the filters were counted on a Zeiss Axioscope.

\section{Statistics}

Data was analyzed by a statistician in the Department of Statistics at The University of Delaware. A One-way-ANOVA analysis was used with Bonferroni's post hoc analysis for comparison between multiple groups. A student's $t$-test was used for comparison between two groups. Significance was defined as a $P<0.05$.

\section{RESULTS}

To determine whether the actin-associated protein, PCNT1 is preferentially expressed in PCa bone metastases, cDNA microarray analysis of skeletal versus adrenal metastases from three independent patients obtained from the Rapid Autopsy Program at the University of Michigan was performed. The analysis revealed significant upregulation of the PCNT1 (FROUNT) gene in the skeletal versus adrenal metastasis (Fig. 1A). A search of the Oncomine ${ }^{\mathrm{TM}}$ database revealed upregulation of PCNT1 expression in advanced/metastatic $\mathrm{PCa}$ in three independent cDNA microarray experiments (Fig. 1B) [www. oncomine.com].

In order to investigate the contribution of PCNT1 upregulation on PCa metastasis, we assessed the differential expression of PCNT1 in a panel of PCa cells lines by qRT-PCR. The analysis demonstrates that PCNT1 is expressed ubiquitously throughout the panel PCa cells, with PC-3 cells demonstrating significantly higher PCNT1 mRNA expression compared to all other cell lines $(P<0.001 ;$ Fig. $2 \mathrm{~A})$. Recently, monocyte migration was shown to be regulated by activation of CCR2-mediated PCNT1 localization to the lamellipodial protrusions during chemotaxis [Terashima et al., 2005]. To begin to test the role of PCNT1, PC-3 cells were stimulated with increasing concentrations of CCL2, 1-100 ng/ml for $24 \mathrm{~h}$, and the expression of PCNT1 mRNA measured by qRT-PCR. Stimulation of PC-3 cells with CCL2 resulted in a dose-dependent increase in PCNT1 mRNA expression compared to the untreated control $\left({ }^{*} P<0.001\right) \quad$ (Fig. 2B). This suggests that PCNT1 expression may be directly regulated by CCL2 stimulation in PCa cells.

To determine whether CCL2 stimulation of PCa cells results in cytoskeletal changes, PC-3 cells were treated with CCL2 [100 ng/ml for $24 \mathrm{~h}$ ] and then stained for actin organization and PCNT1 localization. Published data suggests that CCL2 stimulation of monocytes induces PCNT1 translocation to the CCR2 receptor, which clustered at the leading edge of migrating cell [Terashima et al., 2005]. Untreated PC-3 cells have a distinct actin organization that consists of thick actin bundles with essentially no organized protrusions (Fig. 3A). In addition, untreated PC-3 cells displayed focal, punctate staining of PCNT1 that was primarily perinuclear (Fig. 3B). Stimulation of PC-3 with CCL2 
A
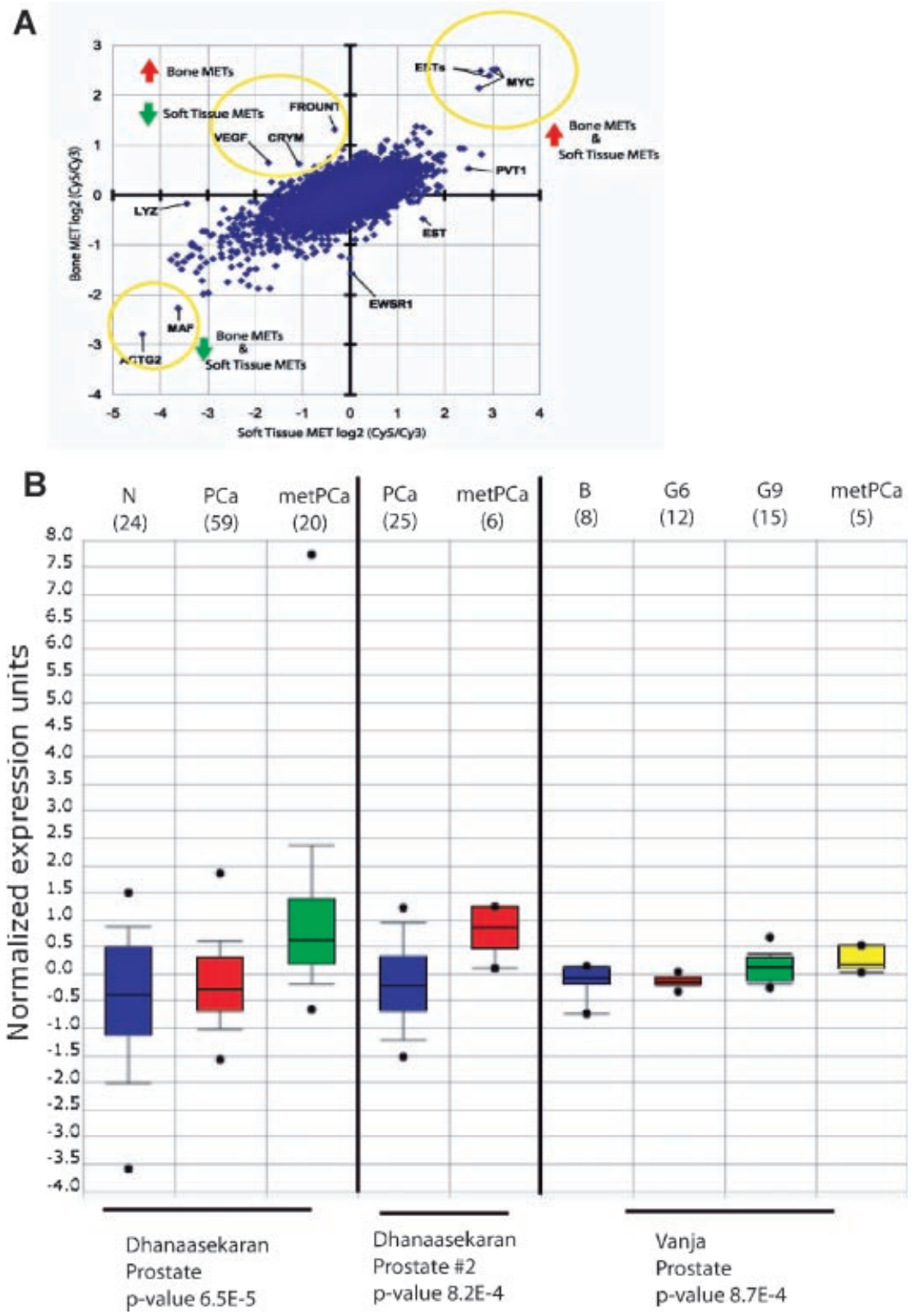

Fig. 1. cDNA microarray analysis identifies FROUNT ( $p C N T 1$ ) as significant in prostate cancer bone metastasis. A: The results from a cDNA microarray comparison between prostate cancer bone metastases and soft tissue (adrenal) metastases from a single patient are shown. Similarly expressed genes remain around the $(0,0)$ intersection and extend along a diagonal rising from left to right. Genes that are differentially expressed appear in the upper left and lower right quadrants of the plot. Select genes are marked for reference. Data from a $20 \mathrm{~K}$ cDNA microarray was $\log 2$

significantly altered the actin morphology and resulted in lamellipodia formation and filopodial protrusions (arrow; Fig. 3D) and a more diffuse cytoplasmic staining of PCNT1 (Fig. 3E). Taken together these data suggest that CCL2induced chemotaxis of $\mathrm{PCa}$ cells may, in part, be transformed and median centered. B: Upregulation of PCNT1 in advanced prostate cancer was further identified in three independent cDNA microarray analyses by mining the Oncomine Database (www.oncomine.com) $(\mathrm{N}=$ normal; $\mathrm{B}=$ benign prostatic hyperplasia; $\mathrm{G} 6=$ Gleason score $6 ; \mathrm{G} 9=$ Gleason score $9 ; \mathrm{PCa}=$ prostate cancer; metPCa $=$ metastatic prostate cancer). The number of patients/specimens analyzed in each category is represented in parentheses.

regulated by a direct link via the CCL2 receptor (CCR2) to the actin cytoskeleton and may prove to be a potential therapeutic target for inhibiting PCa cell migration and metastasis.

The Rho subfamily of monomeric GTPases play a significant role in reorganizing the actin 
A

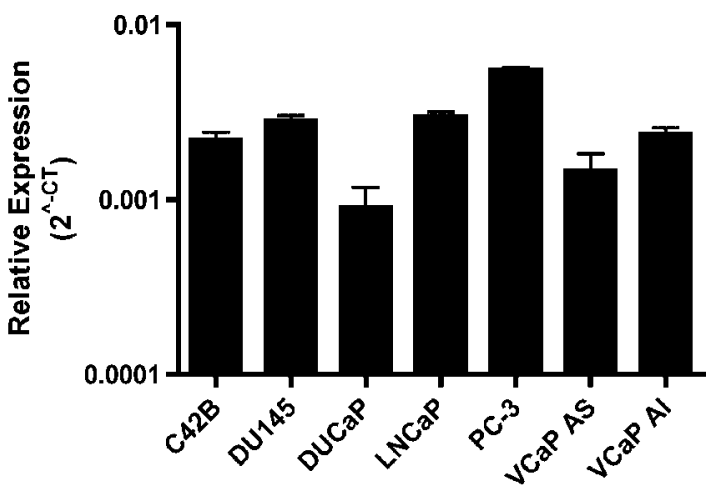

B

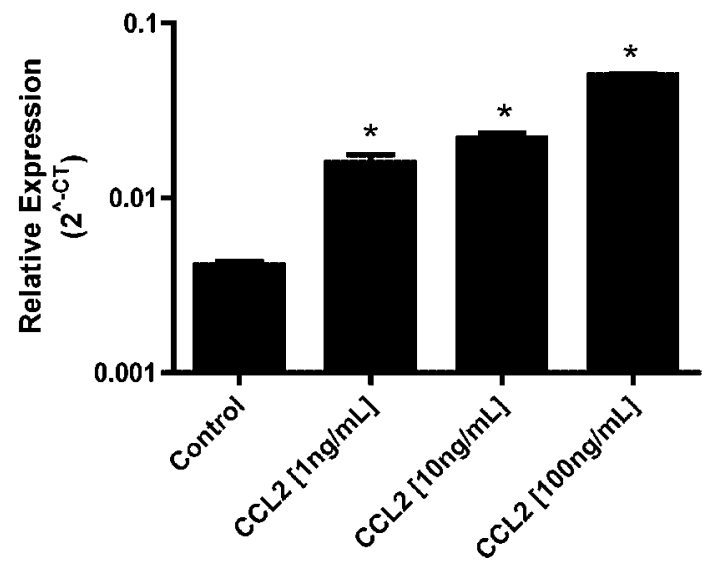

Fig. 2. Expression of PCNT in prostate cancer cells is induced by CCL2 stimulation. Panel A: PCNT1 mRNA expression was assessed by real time-PCR in a panel of prostate cancer cell lines (VCaP AS, androgen sensitive prostate cancer cell line; VCaP AI, androgen independent daughter prostate cancer cell line of $\mathrm{VCaP}$ AS). Relative expression to internal GAPDH expression is displayed as mean $\pm S D$ from three independent experiments performed in replicates of three. Panel B: PC-3 prostate cancer cells were serum starved for $16 \mathrm{~h}$ prior to stimulation with human recombinant CCL2 [1-100 ng/ml] for $24 \mathrm{~h}$ in serum free media. PCNT1 expression was assessed by real time-PCR. Relative expression to internal GAPDH expression is displayed as mean \pm SD from three independent experiments performed in replicates of three $\left({ }^{*} P<0.01\right)$.

cytoskeleton by translating signals from the outside environment [Ridley and Hall, 1992a]. The small GTPase Rac has been shown to actively form growth-factor induced lamellipodia in cells [Ridley and Hall, 1992b]. Previously, CCL2 was shown to induce translocation of PCNT1 in THP-1 monocytes leading to Rac activation [Terashima et al., 2005]. Additionally, we have previously shown that stimulation of PC-3 cells with CCL2 induces actin cytoskeletal rearrangement and the induction of lamellipodial formation [Loberg et al., 2006]. To determine if CCL2 stimulation of PC-3 induces
Rac activation, PC-3 cells were either left unstimulated or stimulated with $100 \mathrm{ng} / \mathrm{ml}$ CCL2 for 30 min and a GLISA Rac activation assay performed. Data from three individual analyses are shown in Figure 4A and demonstrate a significant 3.1-fold increase in active Rac GTPase levels $(P<0.005)$ in the CCL2 stimulated cells compared to the unstimulated cells.

To determine the relative levels of total and active Rac; a time course of Rac activation and expression was performed from 0 to $30 \mathrm{~min}$. using the previously described GST-PAK pulldown assay [Yao et al., 2006]. Protein lysates were collected, and active GTP-bound Rac precipitated with a GST-PAK-sepharose conjugate. The precipitate was collected by centrifugation, resuspended in Laemelli buffer, separated by SDS-PAGE and immunoblotting performed with a Rac-specific antibody. Total GDP- and GTP-bound Rac was detected by immunoblotting of whole cell lysates Figure 4B. Densitometry was performed as described above and the ratio of active GTP-bound Rac to total Rac determined. The results of triplicate assays were combined and are described as relative levels of Rac activation (Fig. 4C). Stimulation of PC-3 cells with CCL2 induced a 2.4-fold increase in Rac activation over a 30 min time course; suggesting that a potential mechanism of CCL2-mediated actin cytoskeletal rearrangement is dependent upon Rac activation. Rac is known to play a prominent role in the initial phase of cell migration and development of the leading edge and formation of lamellipodia [Nobes and Hall, 1995]. Additionally, Rac has been shown to actively recruit $\alpha v \beta 3$ integrins to the leading edge of a migrating cell as a mechanism that promotes formation of new adhesive interactions with the basement membrane [Kiosses et al., 2001].

We next tested whether Rac is activated through PCNT1 and in turn contributes to the ability of PCa cells to interact with HBME cells undergo transendothelial cell migration. PC-3 cells were transiently transfected with three independent siRNA constructs specifically targeting PCNT1. Immunoblot analysis confirmed a 54-96\% decrease in PCNT1 protein expression using all three siRNA constructs compared to the scrambled siRNA control (Fig. 5A).

Next, a GLISA Rac activation assay was performed on PC-3 cells transiently transfected with either the scrambled siRNA control or a 


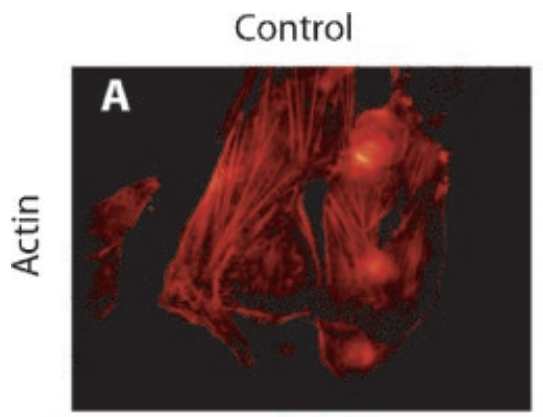

CCL2 (100 ng/mL, $24 \mathrm{hrs})$
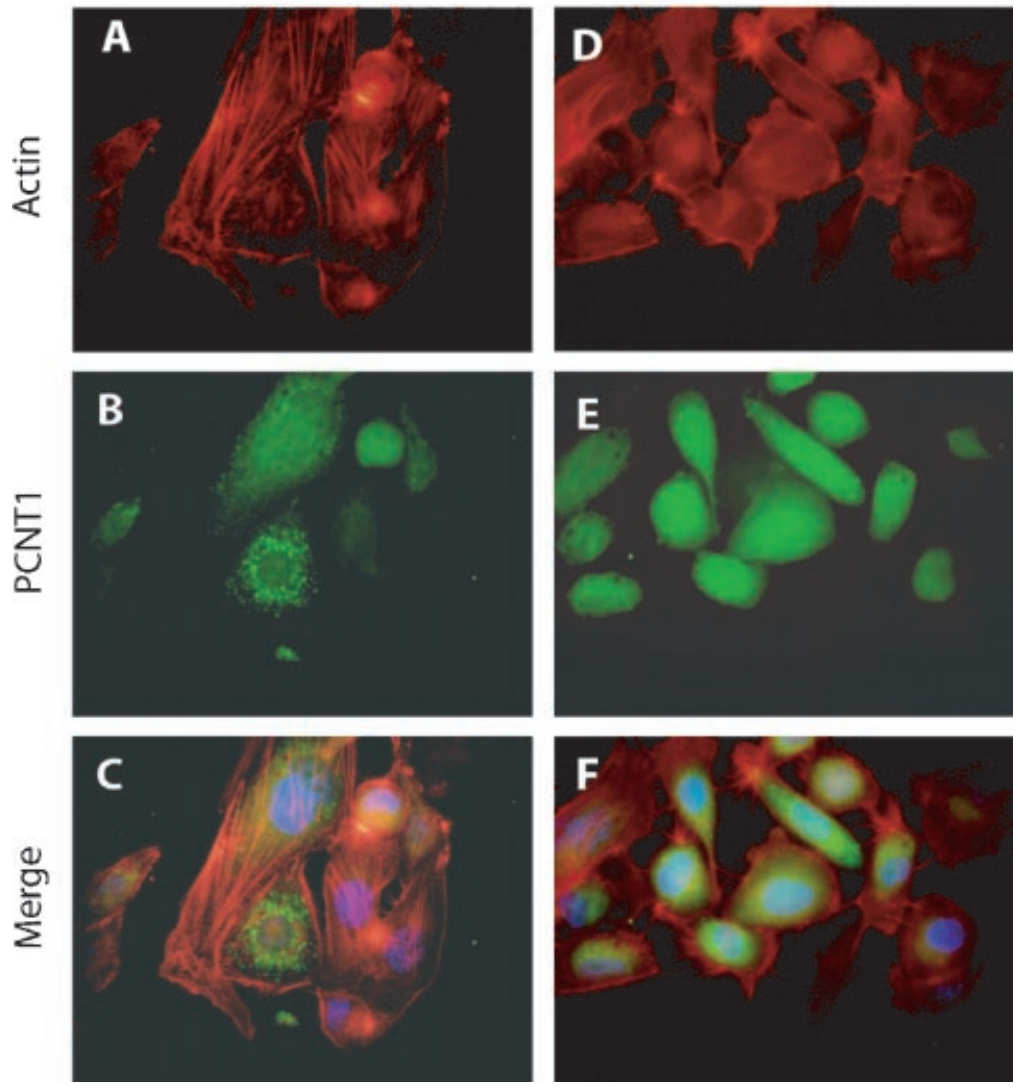

Fig. 3. Stimulation of PC-3 cells with CCL2 induces PCNT1 redistribution and actin cytoskeletal rearrangement. PC-3 cells were treated with CCL2 $(100 \mathrm{ng} / \mathrm{ml}$ for $24 \mathrm{~h}$ in serum free media] and stained for actin (red) organization and PCNT1 (green) localization. Representative figures are displayed demonstrating actin rearrangement in CCL2 treated cells $(\mathbf{D})$ compared to untreated controls $(\mathbf{A})$. In untreated cells PCNT1 was found to be localized in punctuate perineuclear clusters (B) that dissociated and became diffuse throughout the cytoplasm with CCL2 treatment $(\mathbf{E})$. The merge of actin and PCNT1 staining is shown $(\mathbf{C}, \mathbf{F})$.

pool of the three siRNAs to PCNT1 or Rac GTPase and stimulated with CCL2 $(100 \mathrm{ng} / \mathrm{ml})$ for $30 \mathrm{~min}$. As shown in Figure 5B, Rac activation in PC-3 cells expressing siRNA to PCNT1 was significantly decreased compared with the control transfectants $(P<0.0043)$, suggesting that in PC-3 cells, Rac GTPase is directly activated by CCL2 via PCNT1. Direct downregulation of Rac GTPase, shown in Figure 5B(ii), led to a nearly $80 \%$ decrease in Rac activation after CCL2 stimulation $(P<0.001)$. This demonstrates that inhibition of CCL2-stimulated Rac activation is similar when either PCNT1 expression is inhibited or Rac expression is directly downregulated.

The effect of inhibiting PCNT1 and thus, Rac activation, on binding of $\mathrm{PCa}$ cells to $\mathrm{HBME}$ cells was tested (Fig. 5C). Binding of PC-3 cells expressing either siRNA to PCNT1 or to Rac
GTPases decreased the cells ability to bind to HBME cells by $20-40 \%$. Although this was not significant, the differences approached significance. Suggesting that activation of Rac GTPase through CCL2 stimulation contributes to the ability of the PCa cells to bind to and interact with HBME cells.

To determine the role of PCNT1 in PCa transendothelial cell migration we used the PCNT1 siRNA transfected PC-3 cells and assessed their ability to undergo tumor diapedesis in vitro. First, HBME cells were grown to confluence Matrigel in a transwell insert and cells were allowed to invade for $24 \mathrm{~h}$. Inhibition of PCNT1 significantly reduced the ability of PC-3 cells to migrate though the HBME/ Matrigel layer by 50-80\% (Fig. 5D). Taken together these data provide evidence for the role of CCL2 mediating PCa cell binding to and 
A

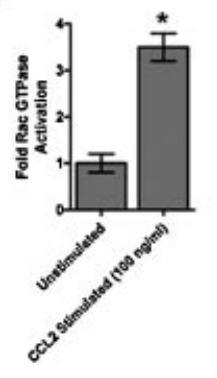

B

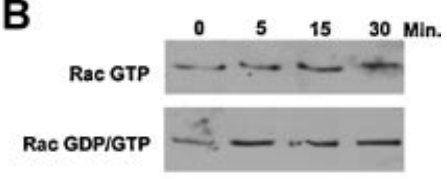

C

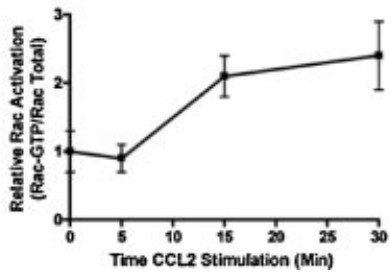

Fig. 4. Activation of Rac GTPase in PC-3 prostate cancer cells after stimulation with CCL2. Panel A: PC-3 cells were stimulated for $30 \mathrm{~min}$ at $37^{\circ} \mathrm{C}$ with $100 \mathrm{ng} / \mathrm{ml} \mathrm{CCL2}$ and a GLISA Rac GTPase activation assay performed. Results from three independent experiments demonstrate that active GTP-bound Rac levels were significantly higher, $* P<0.005$, in the stimulated cells compared with unstimulated PC-3 cells. Relative levels of active versus total Rac GTPase were measured in PC-3 over a 30 min time course after CCL2 stimulation using a GST-PAK pull down. Panel B is a representative matched immunoblot of active Rac GTP and total Rac GDP/GTP. Both active and total levels appear to increase over the 30 min following CCL2 stimulation. Panel C is the relative levels of active Rac over the 30 min time course of CCL2 activation. Densitometry was performed on the active and total Rac immunoblots and a ratio of active and total Rac determined. Quantitation is the result of three independent experiments.

transendothelial cell migration of HBME cells through PCNT1 activation of Rac GTPase.

\section{DISCUSSION}

PCNT1 or pericentrin appears to be of particular interest for a number of reasons [Pihan et al., 2001]. Cellular architecture and genomic stability are controlled in part by centrosomes; organelles that organize microtubule arrays including mitotic spindles. Pihan et al. [2001] have demonstrated that centrosomes are structurally and numerically abnormal in the majority of metastatic prostate carcinomas. They demonstrated that centrosome abnormalities increase with increasing Gleason grade and with increasing levels of genomic instability and that selective induction of centrosome abnormalities by elevating levels of the centrosome protein pericentrin in prostate epithelial cell lines reproduced many of the phenotypic characteristics of high-grade prostate carcinoma. Pericentrin also has another interesting property that may be particularly

relevant to $\mathrm{PCa}$ metastasis to bone. It has several helix-sheet-coil domains and has a sequence homologous to chitanase. Chitinases were originally described as a family of proteins that dissolve hard chitin shells found in the lower phyla [Connor et al., 2000; Fusetti et al., 2002; Recklies et al., 2002]. Recently, a human chitotriosidase was described as a marker for Gaucher disease with plasma levels of the enzyme elevated up to two orders of magnitude [Fusetti et al., 2002]. Inactivation of this enzyme is thought to be responsible for the significant changes in bone metabolism and fat deposition observed in that disease [Fusetti et al., 2002]. Fusetti et al. [2002] have been able to demonstrate how the chitinases have evolved into mammalian lectins such as human cartilage glycoprotein-39 (HC gp-39) by the mutation of key residues in the active site, tuning the substrate binding specificity. HC gp-39 appears to play a significant role in inflammatory arthritis conditions where it is elevated in the synovial fluid [Connor et al., 2000; Recklies et al., 2002]. Furthermore, osteoblasts at sites of endochondral and intramembranous bone formation were positive for expression of $\mathrm{HC}$ gp-39 [Connor et al., 2000]. Taken together, these data suggest that PCNT1 is elevated in metastatic $\mathrm{PCa}$ and has similarity to other enzymes that are active in extracellular matrix remodeling.

The role of the GTPases RhoA, RhoC, and Rac in PCa biology is just beginning to be elucidated, particularly in the area of bone metastasis. To date, few studies have attempted to define the Rho GTPase profile of metastatic PCa cells. A recent study by Hodge et al. [2003] suggests that increased RhoA expression and activity are responsible for NF- $\mathrm{KB}$-mediated PC-3 cell invasion. $\mathrm{NF}-\kappa \mathrm{B}$ is known to be an important transcription factor in regulating the synthesis of CCL2 [Kim et al., 2005]. RhoA is known to inhibit invasion and transendothelial cell migration and to induce stress fiber formation throughout the cell. We have shown that activation of RhoC GTPase promotes invasion and directional migration in $\mathrm{PCa}$ cells [Hall, 1990; Xin et al., 2003; Lin et al., 2005]. Rac activation is known to promote linear movement and diapedesis via the induction of actin cytoskeletal reorganization and promoting filipodial formation. The data presented here demonstrates that stimulation of PCa cells with CCL2 induces transendothelial cell migration that is dependent upon activation of Rac and 
A

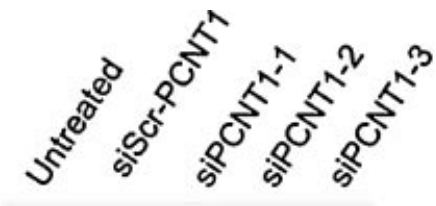

PCNT1

Actin

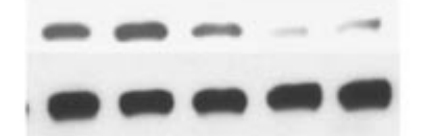

B i.

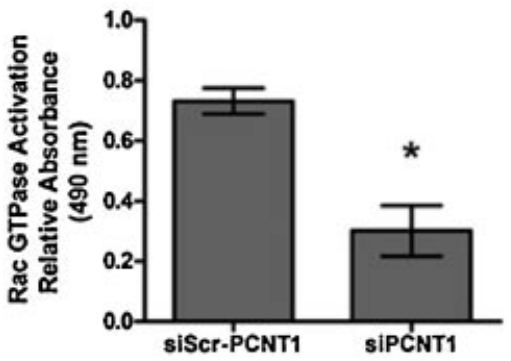

C

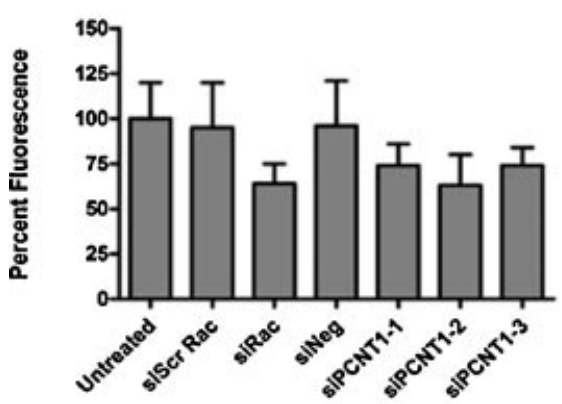

Fig. 5. PCNT1 activation of Rac GTPase. Panel $\mathbf{A}$ is a Western blot analysis of PCNT1 expression in PC-3 cells transfected with PCNT1-specific siRNAs or scrambled siRNA control. Expression of PCNT1 was decreased by all three siRNAs to PCNT1 compared with the scrambled control. Panel B: (i) Rac activation in PC-3 cells transfected with a pool of three PCNT1 siRNAs or scrambled control. siRNA transfected PC-3 cells were stimulated with $100 \mathrm{ng} / \mathrm{ml} \mathrm{CCL2}$ for $30 \mathrm{~min}$ and Rac activation measured using the GLISA assay. Results from triplicate experiments demonstrate a significant decrease $\left({ }^{*} P<0.0043\right)$ in Rac activation in the siPCNT1-treated cells compared with the scrambled control. These results were similar to what was observed when Rac was directly downregulated by siRNA (ii) and PC-3 cells

interactions with PCNT1 resulting in cytoskeletal reorganization.

Previous work in our laboratory has demonstrated that Rac is an important mediator of ii.

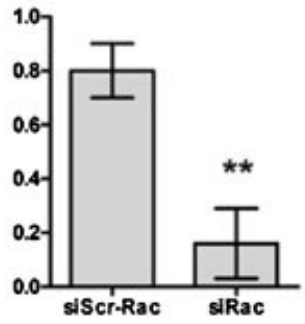

D

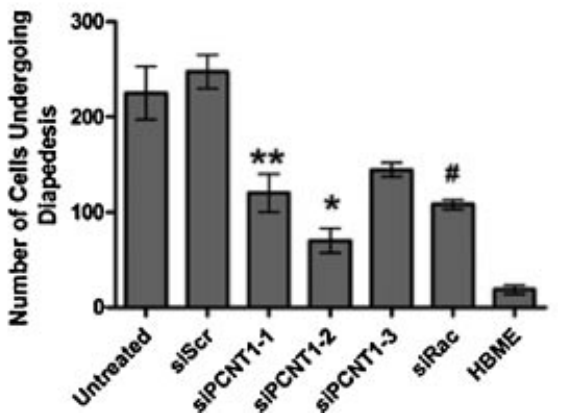

stimulated with CCL2. Direct downregulation of Rac resulted in an $80 \%$ decrease $(* * P<0.001)$ in Rac activation. Panel $\mathbf{C}$ is a binding assay of PC-3 cells to human bone marrow endothelial cells. The ability of the PC-3 cells to adhere to HBME cells after inhibition of Rac GTPase or PCNT1 was slightly decreased but was not significant. Panel $\mathbf{D}$ are the results of a transendothelial cell migration assay. Introduction of siRNA to Rac significantly decreased the PC-3 cells ability to undergo tumor cell diapedesis $\left({ }^{\#} P<0.003\right)$. Similarly, two of the individual siRNAs to PCNT1 significantly reduced the PC-3 cells ability to cross the endothelial cell layer $\left({ }^{*} P<0.0001\right.$ and $\left.{ }^{* *} P<0.0032\right)$. All analyses were performed in triplicate.

transendothelial migration of PC-3 cells [Yao et al., 2006]. To delineate the role of Rac in invasion versus transendothelial cell migration we employed the stably siRNA transfected PC-3 
cells in a modified invasion assay. HBME cells were added to the upper chamber of a transwell insert containing $8 \mu \mathrm{m}$ pores. Serum containing media was placed in the lower chamber on the day of the assay. PC-3 cells were then placed in the upper chamber in serum free media for $24 \mathrm{~h}$. Targeted disruption of Rac attenuated the transendothelial cell migration of PC-3 cells.

Taken together, these data suggest that CCL2 stimulation of $\mathrm{PCa}$ cells induces an invasive phenotype that is dependent on PCNT1 upregulation and Rac activation and are important in the regulation of transendothelial cell migration of PCa cells.

\section{ACKNOWLEDGMENTS}

We thank the members of the Center for Translational Cancer Research at The University of Delaware for insightful discussions. We also thank Dr. Harry Yao for his technical expertise. This project was funded in part by University of Michigan Prostate SPORE Career Development Award P50 CA69568-06A (RDL), PO1 CA093900-01 (RDL, KJP), and CDMRP PCRP W81XH-04-0225, W81XH-05-0005 and The University of Delaware/Delaware Biotechnology Institute Start Up funds (KvG).

\section{REFERENCES}

Balkwill F. 2003. Chemokine biology in cancer. Semin Immunol 15:49-55.

Connor JR, Dodds RA, Emery JG, Kirkpatrick RB, Rosenberg M, Gowen M. 2000. Human cartilage glycoprotein 39 (HC gp-39) mRNA expression in adult and fetal chondrocytes, osteoblasts and osteocytes by in-situ hybridization. Osteoarthritis Cartilage 8:87-95.

Cooper CR, Pienta KJ. 2000. Cell adhesion and chemotaxis in prostate cancer metastasis to bone: A minireview. Prostate Cancer Prostatic Dis 3:6-12.

Cooper CR, McLean L, Walsh M, Taylor J, Hayasaka S, Bhatia J, Pienta KJ. 2000. Preferential adhesion of prostate cancer cells to bone is mediated by binding to bone marrow endothelial cells as compared to extracellular matrix components in vitro. Clin Cancer Res 6: 4839-4847.

Esteve P, Embade N, Perona R, Jiménez B, del Peso L, León J, Arends M, Miki T, Lacal JC. 1998. Rho-regulated signals induce apoptosis in vitro and in vivo by a p53independent, but Bcl2 dependent pathway. Oncogene 17: $1855-1869$

Evers EE, van der Kammen RA, ten Klooster JP, Collard JG. 2000. Rho-like GTPases in tumor cell invasion. Methods Enzymol 325:403-415.

Fidler IJ. 1990. Critical factors in the biology of human cancer metastasis: Twenty-eighth G.H.A. Clowes memorial award lecture. Cancer Res 50:6130-6138.
Fusetti F, von Moeller H, Houston D, Rozeboom HJ, Dijkstra BW, Boot RG, Aerts JM, van Aalten DM. 2002. Structure of human chitotriosidase. Implications for specific inhibitor design and function of mammalian chitinase-like lectins. J Biol Chem 277:25537-25544.

Gampel A, Parker PJ, Mellor H. 1999. Regulation of epidermal growth factor receptor traffic by the small GTPase rhoB. Curr Biol 9:955-958.

Hall A. 1990. The cellular functions of small GTP-binding proteins. Science 249:635-640.

Hall CL, Dai J, van Golen KL, Keller ET, Long MW. 2006. Type I Collagen Receptor (\{alpha\}2\{beta\}1) Signaling Promotes the Growth of Human Prostate Cancer Cells within the Bone. Cancer Res 66:8648-8654.

Hodge JC, Bub J, Kaul S, Kajdacsy-Balla A, Lindholm PF. 2003. Requirement of RhoA activity for increased nuclear factor kappaB activity and PC-3 human prostate cancer cell invasion. Cancer Res 63:1359-1364.

Kim MS, Day CJ, Morrison NA. 2005. MCP-1 is induced by receptor activator of nuclear factor-\{kappa $\} \mathrm{B}$ ligand, promotes human osteoclast fusion, and rescues granulocyte macrophage colony-stimulating factor suppression of osteoclast formation. J Biol Chem 280:16163-16169.

Kiosses WB, Shattil SJ, Pampori N, Schwartz MA. 2001. Rac recruits high-affinity integrin alphavbeta3 to lamellipodia in endothelial cell migration. Nat Cell Biol 3:316320 .

Lehr JE, Pienta KJ. 1998. Preferential adhesion of prostate cancer cells to a human bone marrow endothelial cell line. J Natl Cancer Inst 90:118-123.

Lin M, Van Golen CM, Van Golen KL. 2005. RhoC and Rac1 GTPases in prostate cancer cell motility, invasion, and metastasis. AACR Meeting Abstracts, Apr 2005:239-240.

Loberg RD, Day LL, Harwood J, Ying C, St John LN, Giles R, Neeley CK, Pienta KJ. 2006. CCL2 is a potent regulator of prostate cancer cell migration and proliferation. Neoplasia 8:578-586.

Loberg RD, Tantivejkul K, Craig M, Neeley CK, Pienta KJ. 2007. PAR1-mediated RhoA activation facilitates CCL2induced chemotaxis in PC-3 cells. J Cell Biochem 101: 1292-1300.

Nobes CD, Hall A. 1995. Rho, rac, and cdc42 GTPases regulate the assembly of multimolecular focal complexes associated with actin stress fibers, lamellipodia, and filopodia. Cell 81:53-62.

Ohta M, Kitadai Y, Tanaka S, Yoshihara M, Yasui W, Mukaida N, Haruma K, Chayama K. 2002. Monocyte chemoattractant protein-1 expression correlates with macrophage infiltration and tumor vascularity in human esophageal squamous cell carcinomas. Int J Cancer 102: 220-224.

Pihan GA, Purohit A, Wallace J, Malhotra R, Liotta L, Doxsey SJ. 2001. Centrosome defects can account for cellular and genetic changes that characterize prostate cancer progression. Cancer Res 61:2212-2219.

Recklies AD, White C, Ling H. 2002. The chitinase 3-like protein human cartilage glycoprotein 39 (HC-gp39) stimulates proliferation of human connective-tissue cells and activates both extracellular signal-regulated kinaseand protein kinase B-mediated signalling pathways. Biochem J 365:119-126.

Ridley AJ, Hall A. 1992a. The small GTP-binding protein rac regulates growth factor-induced membrane ruffling. Cell 70:401-410. 
Ridley AJ, Hall A. 1992b. Distinct patterns of actin organization regulated by the small GTP-binding proteins Rac and Rho. Cold Spring Harb Symp Quant Biol 57:661-671.

Romanov VI, Goligorsky MS. 1999. RGD-recognizing integrins mediate interactions of human prostate carcinoma cells with endothelial cells in vitro. Prostate 39: 108-118.

Sahai E, Marshall CJ. 2002. RHO-GTPases and cancer. Nat Rev Cancer 2:133-142.

Schweitzer KM, Vicart P, Delouis C, Paulin D, Dräger AM, Langenhuijsen MM, Weksler BB. 1997. Characterization of a newly established human bone marrow endothelial cell line: Distinct adhesive properties for hematopoietic progenitors compared with human umbilical vein endothelial cells. Lab Invest 76:25-36.

Terashima Y, Onai N, Murai M, Enomoto M, Poonpiriya V, Hamada T, Motomura K, Suwa M, Ezaki T, Haga T,
Kanegasaki S, Matsushima K. 2005. Pivotal function for cytoplasmic protein FROUNT in CCR2-mediated monocyte chemotaxis. Nat Immunol 6:827-835.

Thalmann GN, Sikes RA, Wu TT, Degeorges A, Chang SM, Ozen M, Pathak S, Chung LW. 2000. LNCaP progression model of human prostate cancer: Androgenindependence and osseous metastasis. Prostate 44:91103.

van Golen KL. 2003. Inflammatory breast cancer: Relationship between growth factor signaling and motility in aggressive cancers. Breast Cancer Res 5:174-179.

Xin W, Rhoses DR, Ingold C, Chinnaiyan AM, Rubin MA. 2003. Dysregulation of the annexin family protein family is associated with prostate cancer progression. Am J Pathol 162:255-261.

Yao H, Dashner EJ, van Golen CM, van Golen KL. 2006. RhoC GTPase is required for PC-3 prostate cancer cell invasion but not motility. Oncogene 25:2285-2296. 